\title{
Generalised prime systems with periodic integer counting function
}

Article

Accepted Version

Hilberdink, T. (2012) Generalised prime systems with periodic integer counting function. Acta Arithmetica, 152 (3). pp. 217241. ISSN 1730-6264 doi: https://doi.org/10.4064/aa152-3-1 Available at https://centaur.reading.ac.uk/23409/

It is advisable to refer to the publisher's version if you intend to cite from the work. See Guidance on citing.

To link to this article DOI: http://dx.doi.org/10.4064/aa152-3-1

Publisher: Institutum Mathematicum - Academia Scientiarum Polona

All outputs in CentAUR are protected by Intellectual Property Rights law, including copyright law. Copyright and IPR is retained by the creators or other copyright holders. Terms and conditions for use of this material are defined in the End User Agreement.

\section{www.reading.ac.uk/centaur}

\section{CentAUR}

Central Archive at the University of Reading

Reading's research outputs online 


\title{
Generalised prime systems with periodic integer counting function ${ }^{1}$
}

\author{
Titus Hilberdink \\ Department of Mathematics, University of Reading, Whiteknights, \\ PO Box 220, Reading RG6 6AX, UK; t.w.hilberdink@reading.ac.uk
}

\begin{abstract}
We study generalised prime systems (both discrete and continuous) for which the 'integer counting function' $N(x)$ has the property that $N(x)-c x$ is periodic for some $c>0$. We show that this is extremely rare. In particular, we show that the only such system for which $N$ is continuous is the trivial system with $N(x)-c x$ constant, while if $N$ has finitely many discontinuities per bounded interval, then $N$ must be the counting function of the g-prime system containing the usual primes except for finitely many.
\end{abstract}

2010 AMS Mathematics Subject Classification: 11N80

Keywords and phrases: Generalised prime systems.

\section{Introduction}

In a recent paper [7], we discussed Mellin transforms $\hat{N}(s)$ of integrators $N$ for which $N(x)-x$ is periodic in order to study flows of holomorphic functions converging to $\zeta(s)$. Here we consider the question when such an $N$ determines a g-prime system; i.e. that $N(x)$ is the 'integer counting function' of a generalised prime system — see section 1.3 for the definition.

An example of such a flow $\hat{N}_{\lambda}(s)$ was given (in [7]) but it was unclear whether or not they determined g-prime systems. As a consequence of our results, we show that none of them does.

In fact, we investigate more generally when an increasing function $N$ for which $N(x)-c x$ is periodic determines a g-prime system for a constant $c>0$. (At the outset we assume that $N$ is right-continuous, $N(1)=1$, and $N(x)=0$ for $x<1$.) For example, $N(x)=c x+1-c$ for $x \geq 1$ determines a continuous g-prime system for $0<c \leq 2$ at least.

As for discontinuous examples, we have the prototype $N(x)=[x]$ for the usual primes and integers. For other examples, consider the g-prime system containing the usual primes except given primes $p_{1}, \ldots p_{k}$. This has integer counting function

$$
N(x)=\sum_{\substack{n \leq P \\(n, P)=1}}\left[\frac{x-n}{P}+1\right]
$$

where $P=p_{1} p_{2} \ldots p_{k}$. In this case $N(x+P)=N(x)+\varphi(P)$ where $\varphi$ is Euler's function, and $N(x)-\frac{\varphi(P)}{P} x$ has period $P$.

Our results split quite naturally into continuous and discontinuous cases. In section 2, where we consider the continuous case, the main result is that for $N$ sufficiently 'nice' (eg. continuously differentiable), $N$ determines a g-prime system only for the trivial case where $N(x)-c x$ is constant; i.e. $N(x)=c x+1-c$.

For discontinuous $N$ the picture is less straightforward. A useful tool is to consider its 'jump' function $N_{J}$, which must necessarily also have $N_{J}(x)-c^{\prime} x$ periodic (for some $c^{\prime}>0$ ) and which also determines a g-prime system if $N$ does (Theorem 1.1). We show that if such an $N$ has only finitely many discontinuities in any interval but is otherwise 'smooth', then $N$ must be a step function, the discontinuities must occur at integer points and the period, say $P$, must be a natural number. Then, denoting the jump at $n$ by $a_{n}$, we show that $a_{n}$ is even ${ }^{2}(\bmod P)$ and multiplicative. This allows us to deduce our main result.

\footnotetext{
${ }^{1}$ To appear in Acta Arithmetica.

${ }^{2}$ That is; $a_{n}=a_{(n, P)}$.
} 


\section{Theorem A}

Let $N \in T$ be such that $N(x)$ - cx has period $P$, and suppose that $N$ determines a g-prime system. Then $P \in \mathbb{N}$ and

$$
N(x)=\sum_{\substack{n \leq P \\(n, P)=1}}\left[\frac{x-n}{P}+1\right] .
$$

i.e. $N$ is the integer-counting function of the g-prime system $\mathbb{P} \backslash\left\{p_{1}, \ldots, p_{k}\right\}$ where $p_{1}, \ldots, p_{k}$ are the prime divisors of $P$.

(For the definition of $T$, see section 1.2.) This actually shows that the smallest period must be squarefree and that $c=\frac{\varphi(P)}{P}$. Our set up includes all the usual 'discrete' g-prime systems.

In proving Theorem A, we prove the following result on Dirichlet series with periodic coefficients, which may be of independent interest.

\section{Theorem B}

Let $\left\{a_{n}\right\}_{n \in \mathbb{N}}$ be periodic, $a_{1}=1$, and suppose $a_{n}=\exp _{*} b_{n}$ for some $b_{n} \geq 0$. Then $a_{n}$ is multiplicative.

Here $*$ refers to Dirichlet convolution. Thus $a_{n}$ and $b_{n}$ are related by $\sum_{n=1}^{\infty} \frac{a_{n}}{n^{s}}=\exp \left\{\sum_{n=1}^{\infty} \frac{b_{n}}{n^{s}}\right\}$.

\section{$\S 1$. Preliminaries}

\subsection{Riemann-Stieltjes convolution}

Let $S$ denote the space of functions $f: \mathbb{R} \rightarrow \mathbb{C}$ which are zero on $(-\infty, 1)$, right-continuous, and of local bounded variation. (See e.g. [3], pp.50-70.) This is a vector space over addition. Let $S^{+}$denote the subspace of $S$ consisting of increasing functions. Also, for $\alpha \in \mathbb{R}$, let $S_{\alpha}=\{f \in S: f(1)=\alpha\}$, while $S_{\alpha}^{+}=S^{+} \cap S_{\alpha}$.

For functions $f, g \in S$, define the convolution (or Mellin-Stieltjes convolution) by ${ }^{3}$

$$
(f * g)(x)=\int_{1-}^{x} f\left(\frac{x}{t}\right) d g(t) .
$$

We note that $S$ is closed under $*$ and that $*$ is commutative and associative. The identity (w.r.t. $*$ ) is $i(x)=1$ for $x \geq 1$ and zero otherwise.

(a) If $f$ or $g$ is continuous (on $\mathbb{R}$ ), then $f * g$ is continuous.

(b) Exponentials. For $f \in S_{1}$, there exists $g \in S_{0}$ such that $f=\exp _{*} g$; i.e.

$$
f=\sum_{n=0}^{\infty} \frac{g^{* n}}{n !}
$$

where $g^{* n}=g * g^{*(n-1)}$ and $g^{* 0}=i$. Also $f=\exp _{*} g$ if and only if $f * g_{L}=f_{L}$ (see [5]), where $f_{L} \in S$ is the function defined for $x \geq 1$ by $f_{L}(x)=\int_{1}^{x} \log t d f(t)$.

(c) For $f \in S$, define the Mellin transform of $f$ by $\hat{f}(s)=\int_{1-}^{\infty} x^{-s} d f(x)$. This exists if $f(x)=O\left(x^{A}\right)$ for some $A$. Note that $\widehat{f * g}=\hat{f} \hat{g}$ and $\widehat{\exp _{*} f}=\exp \hat{f}$.

(d) Let $f, g \in S$ be continuously differentiable on $(1, \infty)$. Let $g_{1}(x)=\int_{1-}^{x} \frac{1}{t} d g(t)$. Then $f * g$ is also continuously differentiable on $(1, \infty)$ with

$$
(f * g)^{\prime}=f^{\prime} * g_{1}+f(1) g^{\prime} .
$$

Proof. Let $x>1$ and consider $(f * g)(x+h)-(f * g)(x)$ for $h$ small. Consider $h>0$ first. We have

$$
\frac{(f * g)(x+h)-(f * g)(x)}{h}=\int_{1-}^{x} \frac{f\left(\frac{x+h}{t}\right)-f\left(\frac{x}{t}\right)}{h} d g(t)+\frac{1}{h} \int_{x}^{x+h} f\left(\frac{x+h}{t}\right) d g(t) .
$$

\footnotetext{
${ }^{3}$ All limits of integration are understood to be + (i.e. from the right) except where they are explicitly stated to be -.
} 
The integrand in the first integral tends pointwise to $\frac{1}{t} f^{\prime}\left(\frac{x}{t}\right)$, so by the continuity of $f^{\prime}$ this integral tends to (see [1], p.218)

$$
\int_{1-}^{x} \frac{f^{\prime}\left(\frac{x}{t}\right)}{t} d g(t)=\left(f^{\prime} * g_{1}\right)(x) \quad \text { as } h \rightarrow 0
$$

The second term equals

$$
f(1) \frac{g(x+h)-g(x)}{h}+\frac{1}{h} \int_{x}^{x+h}\left(f\left(\frac{x+h}{t}\right)-f(1)\right) d g(t) .
$$

The first term tends to $f(1) g^{\prime}(x)$ while the integrand tends to 0 by right-continuity of $f$ at 1 . Hence so does the integral.

If $h<0$, write $h=-k$ and split up as $\frac{1}{k} \int_{1}^{x-k}$ and $\frac{1}{k} \int_{x-k}^{x}$ and argue as before.

For the proofs of (a)-(c) see [3] and [5].

\subsection{The 'jump' function}

Definition 1.1: (i) For $f \in S$ and each $x \in \mathbb{R}$, we denote by $\Delta f(x)$ the left-hand jump of $f$ at $x$; i.e.

$$
\Delta f(x)=f(x)-f(x-)=\lim _{h \rightarrow 0^{+}}(f(x)-f(x-h)) .
$$

This is well-defined for monotone $f$ and hence for $f \in S$. Note also that $\Delta f$ is non-zero on a countable set only ([1], p.162).

(ii) For $f \in S^{+}$, let $f_{J}$ denote the jump function of $f$; i.e.

$$
f_{J}(x)=\sum_{x_{r} \leq x} \Delta f\left(x_{r}\right)
$$

where the $x_{r}$ denote the discontinuities of $f$.

The function $f_{J}$ is increasing and $f=f_{J}+f_{C}$, where $f_{C}$ is continuous and increasing ([1], p.186).

Let $\delta_{a}$ denote the function which is 1 on $[a, \infty)$ and zero otherwise. Note that $\delta_{a} * \delta_{b}=\delta_{a b}$. Letting $D_{f}$ denote the (countable) set of discontinuities of $f$, we may write

$$
f_{J}=\sum_{\alpha \in D_{f}} \Delta f(\alpha) \delta_{\alpha}
$$

The series has only non-negative terms and converges absolutely.

Properties. Let $f, g \in S^{+}$.

(a) $(f * g)_{J}=f_{J} * g_{J}$.

Write $f=f_{J}+f_{C}$ and similarly for $g$. Then

$$
f * g=\left(f_{J}+f_{C}\right) *\left(g_{J}+g_{C}\right)=f_{J} * g_{J}+f_{J} * g_{C}+f_{C} * g_{J}+f_{C} * g_{C} .
$$

The last three terms are all continuous, and so their jump functions are identically zero. Therefore we need to show $\left(f_{J} * g_{J}\right)_{J}=f_{J} * g_{J}$.

To see this, use (1.2) for $f_{J}$ and $g_{J}$. Hence

$$
f_{J} * g_{J}=\sum_{\alpha \in D_{f}} \sum_{\beta \in D_{g}} \Delta f(\alpha) \Delta g(\beta) \delta_{\alpha} * \delta_{\beta}=\sum_{\alpha \in D_{f}} \sum_{\beta \in D_{g}} \Delta f(\alpha) \Delta g(\beta) \delta_{\alpha \beta},
$$

which is a sum of the form $\sum_{\gamma} c_{\gamma} \delta_{\gamma}$; i.e. a jump function. Thus $\left(f_{J} * g_{J}\right)_{J}=f_{J} * g_{J}$ as required. 
(b) For $x \geq 1$, we have

$$
\Delta(f * g)(x)=\sum_{\substack{\alpha \beta=x \\ \alpha \in D_{f}, \beta \in D_{g}}} \Delta f(\alpha) \Delta g(\beta) .
$$

Take $\Delta$ of both sides of (1.3). As the last three terms are all continuous, $\Delta=0$ for these functions. For the remaining term

$$
\Delta\left(f_{J} * g_{J}\right)(x)=\sum_{\alpha \in D_{f}, \beta \in D_{g}} \Delta f(\alpha) \Delta g(\beta) \Delta \delta_{\alpha \beta}(x)=\sum_{\substack{\alpha \beta=x \\ \alpha \in D_{f}, \beta \in D_{g}}} \Delta f(\alpha) \Delta g(\beta),
$$

since $\Delta \delta_{a}(x)=1$ for $x=a$ and zero otherwise.

(c) $D_{f * g}=D_{f} D_{g}=\left\{\alpha \beta: \alpha \in D_{f}, \beta \in D_{g}\right\}$.

If $x \notin D_{f} D_{g}$ (i.e. $x \neq \alpha \beta$ for any $\alpha \in D_{f}$ and $\beta \in D_{g}$ ), then there is no contribution to the sum in (1.4). Hence $\Delta(f * g)(x)=0$ and $x \notin D_{f * g}$. Thus $D_{f * g} \subset D_{f} D_{g}$.

For the converse, if $x \in D_{f} D_{g}$ then $x=\alpha \beta$ for some $\alpha \in D_{f}$ and $\beta \in D_{g}$, so that

$$
\Delta(f * g)(x)=\Delta(f * g)(\alpha \beta) \geq \Delta f(\alpha) \Delta g(\beta)>0,
$$

as all the other terms in (1.4) are non-negative. Hence $x \in D_{f * g}$ and $D_{f * g}=D_{f} D_{g}$ follows.

(d) For $f \in S$, let $f_{L}$ denote the function $f_{L}(x)=\int_{1}^{x} \log t d f(t)$. Then $\Delta f_{L}(x)=\Delta f(x) \log x$ (see [3], p.341) and hence $\left(f_{J}\right)_{L}=\left(f_{L}\right)_{J}$. (Both sides equal $\sum_{\alpha \in D_{f}} \Delta f(\alpha) \log \alpha \delta_{\alpha}$.)

The subspace $T$

Consider those functions in $S$ whose right-hand derivative exists and is continuous in $(1, \infty)$; i.e.

$$
f_{+}^{\prime}(x)=\lim _{h \rightarrow 0^{+}} \frac{f(x+h)-f(x)}{h}
$$

exists for each $x>1$ and $f_{+}^{\prime}$ is continuous here. Let $T$ denote the subspace of such functions which have a finite number of discontinuities per bounded interval. For example, all step functions in $S$ lie in $T$ with $f_{+}^{\prime} \equiv 0$. Further for $f \in T, f_{+}^{\prime} \equiv 0$ if and only if $f$ is a step function. This follows from the fact that if $f$ is continuous on an interval, and $f$ has a continuous one sided derivative, then in fact $f^{\prime}$ exists (and of course equals the one-sided derivative) - see [9], p.355. Thus on each interval where $f$ is continuous and $f_{+}^{\prime} \equiv 0$, we must have $f^{\prime} \equiv 0$ so that $f$ is constant here.

Part (d) of 1.1 generalises to functions in $T:$ if $f, g \in T$ then $f * g \in T$ and

$$
(f * g)_{+}^{\prime}=f_{+}^{\prime} * g_{1}+f_{J, 1} * g_{+}^{\prime},
$$

where $g_{1}$ is as before and $f_{J, 1}=\left(f_{J}\right)_{1}$.

Proof. By 1.2(c), $D_{f * g} \subset D_{f} D_{g}$, so $f * g$ has at most finitely many discontinuities per bounded interval.

We have, on $(1, \infty)$,

$$
(f * g)_{+}^{\prime}=\left(f_{J} * g_{J}\right)_{+}^{\prime}+\left(f_{J} * g_{C}\right)_{+}^{\prime}+\left(f_{C} * g_{J}\right)_{+}^{\prime}+\left(f_{C} * g_{C}\right)_{+}^{\prime} .
$$

Now $f_{J} * g_{J}$ is again a step function, so $\left(f_{J} * g_{J}\right)_{+}^{\prime}=0$. Also, $f_{+}^{\prime}=\left(f_{C}\right)_{+}^{\prime}$ so $f_{C}$ is continuously differentiable and similarly for $g_{C}$. By $1.1(\mathrm{~d}),\left(f_{C} * g_{C}\right)_{+}^{\prime}=f_{C}^{\prime} * g_{C, 1}$. For the remaining terms

$$
\left(f_{J} * g_{C}\right)_{+}^{\prime}(x)=\left(\sum_{\alpha \in D_{f}} \Delta f(\alpha) g_{C}\left(\frac{x}{\alpha}\right)\right)_{+}^{\prime}=\sum_{\alpha \in D_{f}} \frac{\Delta f(\alpha)}{\alpha} g_{C}^{\prime}\left(\frac{x}{\alpha}\right) .
$$

This is clear for $x \notin D_{f}$ (since then $\alpha \neq x$ ), but also true if $x \in D_{f}$ since $g_{C}\left(\frac{x}{\alpha}\right)=0$ for $x \leq \alpha$. Thus $\left(f_{J} * g_{C}\right)_{+}^{\prime}=f_{J, 1} * g_{C}^{\prime}$ and similarly $\left(f_{C} * g_{J}\right)_{+}^{\prime}=f_{C}^{\prime} * g_{J, 1}$. Putting these together gives

$$
(f * g)_{+}^{\prime}=f_{J, 1} * g_{C}^{\prime}+f_{C}^{\prime} * g_{J, 1}+f_{C}^{\prime} * g_{C, 1}=f_{J, 1} * g_{+}^{\prime}+f_{+}^{\prime} * g_{1} .
$$


Thus $(f * g)_{+}^{\prime}$ is continuous and $f * g \in T$.

\subsection{Generalized prime systems}

We distinguish between two different types of g-prime system.

Definition 1.2 An outer g-prime system is a pair of functions $\Pi, N$ with $\Pi \in S_{0}^{+}$and $N \in S_{1}^{+}$such that $N=\exp _{*} \Pi$.

Of course, if $\Pi \in S_{0}^{+}$, then $\exp _{*} \Pi \in S_{1}^{+}$, so $\Pi$ determines a g-prime system (with $N=\exp _{*} \Pi$ ). On the other hand if $N \in S_{1}^{+}$, then $N=\exp _{*} \Pi$ for some $\Pi \in S_{0}$ by 1.1(b), but $\Pi$ need not be increasing. If $\Pi$ is increasing, then we say $N$ determines an outer g-prime system. The above definition is somewhat more general than the usual 'generalised primes', since we have not mentioned the equivalent of the prime counting function $\pi(x)$.

Definition 1.3 A g-prime system is an outer g-prime system for which there exists $\pi \in S_{0}^{+}$such that

$$
\Pi(x)=\sum_{k=1}^{\infty} \frac{1}{k} \pi\left(x^{1 / k}\right) .
$$

we say $N$ determines a g-prime system if there exists such an increasing $\pi \in S_{0}$.

Remarks.

(a) As such, $\pi(x)$ is given by

$$
\pi(x)=\sum_{k=1}^{\infty} \frac{\mu(k)}{k} \Pi\left(x^{1 / k}\right) .
$$

In fact this sum always converges for $\Pi \in S^{+}$(since $\Pi\left(x^{1 / k}\right)$ decreases with $k$ and $\sum_{k=1}^{\infty} \frac{\mu(k)}{k}$ converges). But of course $\pi$ need not be increasing.

(b) A g-prime system is discrete if $\pi$ is a step function with integer jumps. In this case the g-primes are the discontinuities of $\pi$ and the step is the multiplicity.

(c) An outer g-prime system is continuous if $N$ (and hence $\Pi$ - see below) is continuous in $(1, \infty)$.

(d) For an outer g-prime system $(\Pi, N)$, let $\psi=\Pi_{L}$ (i.e. $\left.\psi(x)=\int_{1}^{x} \log t d \Pi(t)\right)$ denote the generalised Chebyshev function.

Note that $\psi \in S_{0}^{+}$, and that $N=\exp _{*} \Pi$ is equivalent to $\psi * N=N_{L}$ (see [3] and [5]).

If $N$ determines a g-prime system and $N(x)=c x+O\left(x(\log x)^{-\gamma}\right)$ for some $\gamma>3 / 2$, then by Beurling's Prime Number Theorem ${ }^{4}$ (see [2] or [4]), $\psi(x) \sim x$. Also $\psi_{1}(x)=\log x+\kappa+o(1)$ for some constant $\kappa$, where $\psi_{1}(x)=\int_{1}^{x} \frac{1}{t} d \psi(t)$.

(e) Applying 1.2(c) to outer g-primes shows that $D_{N_{L}}=D_{N} D_{\psi}$. But $D_{N_{L}}=D_{N} \backslash\{1\}$, so $D_{N} \backslash\{1\}=$ $D_{N} D_{\psi}$.

\section{Theorem 1.1}

Let $(\Pi, N)$ be an outer g-prime system. Then

(a) $\Delta \Pi \leq \Delta N$. In particular, $\Pi$ is continuous at the points of continuity of $N$.

(b) $\left(\Pi_{J}, N_{J}\right)$ is an outer g-prime system.

\footnotetext{
${ }^{4}$ This is usually formulated for g-prime systems, but actually proved for outer g-prime systems. No use of $\pi(x)$ being increasing is made, only that of $\Pi(x)$.
} 
Proof. (a) Apply $\Delta$ to both sides of $\psi * N=N_{L}$ and use $\Delta N_{L}(x)=\Delta N(x) \log x$. Thus

$$
\Delta N(x) \log x=\Delta\left(\psi *\left(N_{J}+N_{C}\right)\right)(x)=\Delta\left(\psi * N_{J}\right)(x) \geq \Delta \psi(x),
$$

since $N$ has a jump of 1 at 1 . But $\Delta \psi(x)=\Delta \Pi(x) \log x$, so $\Delta \Pi \leq \Delta N$ and (a) follows.

(b) Take the jump function of both sides of the equation $\psi * N=N_{L}$. Thus $(\psi * N)_{J}=\left(N_{L}\right)_{J}$. By $1.2(\mathrm{a})$ and (d) this is $\psi_{J} * N_{J}=\left(N_{J}\right)_{L}$. Since $N_{J}$ and $\psi_{J}$ are increasing, this implies $\left(\Pi_{J}, N_{J}\right)$ forms a g-prime system.

Theorem 1.1 gives a useful necessary condition for $N \in S_{1}^{+}$to determine a g-prime system; namely that $N_{J}$ must determine a g-prime system. Of course, this is no use if $N$ is continuous, in which case $N_{J}=i$ — the identity w.r.t. *.

Finally, we remark that if $N$ is continuously differentiable on $(1, \infty)$, then so is $\psi$ and $\psi^{\prime}=N_{L}^{\prime}-N^{\prime} * \psi_{1}$. The proof follows 1.1(d) with $f=N$ and $g=\psi$, so that $(f * g)^{\prime}=N_{L}^{\prime}$. The first integral on the RHS of (1.1) then tends to $f^{\prime} * g_{1}=N^{\prime} * \psi_{1}$, while the second integral lies between

$$
\frac{N(1)}{h} \int_{x}^{x+h} d \psi(t) \quad \text { and } \quad \frac{N(1+h)}{h} \int_{x}^{x+h} d \psi(t) .
$$

Since $N$ is right-continuous at 1 , it follows that $\frac{\psi(x+h)-\psi(x)}{h}$, must therefore tend to a limit as $h \rightarrow 0^{+}$. Similarly, for $h \rightarrow 0^{-}$.

In the same way, $N \in T$ implies $\psi \in T$.

\section{§2. Continuous g-prime systems with $N(x)-c x$ periodic.}

Suppose now that $N \in S_{1}$ and $N(x)=c x-R(x)$ where $R(x)$ is periodic for some $c>0$. Extend $R$ to the whole real line by periodicity. Thus $R$ is right continuous, locally of bounded variation, and $R(1)=c-1$.

In what follows we shall always write $N=\exp _{*} \Pi$ where $\Pi \in S_{0}$.

\section{Theorem 2.1}

Let $N(x)=c x-R(x) \in S_{1}^{+}$, where $R$ is continuously differentiable and periodic, and $c>0$. Then $\Pi$ is increasing if and only if $R$ is constant; i.e. $N(x)=c x+1-c$ for $x \geq 1$.

Proof. If $R$ is constant, then $N(x)=c x+1-c(x \geq 1)$ and $\hat{N}(s)=1+\frac{c}{s-1}$. Thus $\hat{\psi}(s)=-\hat{N}^{\prime}(s) / \hat{N}(s)=$ $\frac{1}{s-1}-\frac{1}{s+c-1}$, which implies $\psi^{\prime}(x)=1-x^{-c} \geq 0$. Hence $\Pi$ is increasing.

For the converse, let $R$ be non-constant and suppose, for a contradiction, that $\Pi$ is increasing. Equivalently, suppose that $\psi^{\prime} \geq 0$. Differentiate the relation $N_{L}=\psi * N$, using 1.1(d). Thus for $x>1$,

$$
N^{\prime}(x) \log x=\left(N^{\prime} * \psi_{1}\right)(x)+\psi^{\prime}(x) \geq\left(N^{\prime} * \psi_{1}\right)(x),
$$

where $\psi_{1}(x)=\int_{1}^{x} \frac{1}{t} d \psi(t)$. Since $N^{\prime}=c-R^{\prime}$, this becomes

$$
R^{\prime}(x) \log x-\left(R^{\prime} * \psi_{1}\right)(x) \leq c \log x-c \psi_{1}(x) .
$$

By Beurling's PNT, the righthand side tends to a limit as $x \rightarrow \infty$, so for some constant $A$ and all $x>1$,

$$
R^{\prime}(x) \log x-\left(R^{\prime} * \psi_{1}\right)(x) \leq A
$$

Let $P$ be a period of $R$. Extend $R$ to $\mathbb{R}$ by periodicity. By continuity and periodicity of $R^{\prime}$ there exists $x_{0} \in[0, P]$ such that

$$
R^{\prime}\left(x_{0}\right)=\max _{x \in \mathbb{R}} R^{\prime}(x) .
$$

Furthermore, for $\delta>0$ sufficiently small, the set of points $x$ in $[0, P]$ for which $R^{\prime}(x) \leq R^{\prime}\left(x_{0}\right)-\delta$ contains an interval, say $[\alpha, \beta]$ with $0<\alpha<\beta<P$. (If not then $R^{\prime}$ is constant which forces $R$ constant.) Let 
$x=n P+x_{0}$ in $(2.2)$ where $n \in \mathbb{N}$. Since $\log \left(n P+x_{0}\right)=\psi_{1}\left(n P+x_{0}\right)+O(1)$ and $R^{\prime}$ has period $P,(2.2)$ can be written as

$$
\int_{1-}^{n P+x_{0}} R^{\prime}\left(x_{0}\right)-R^{\prime}\left(P\left\{\frac{n P+x_{0}}{t P}\right\}\right) d \psi_{1}(t) \leq A .
$$

(A different constant $A$.) Note that the integrand is non-negative. Furthermore, the integrand is at least $\delta$ for $t \in\left[\frac{n P+x_{0}}{k P+\beta}, \frac{n P+x_{0}}{k P+\alpha}\right]$ for each positive integer $k \leq n$.

Let $K$ be a fixed positive integer less than $n$. Thus the LHS of (2.3) is at least

$$
\sum_{k=1}^{K} \int_{\frac{n P+x_{0}}{k P+\beta}}^{\frac{n P+x_{0}}{k P+\alpha}} \delta d \psi_{1}(t)=\delta \sum_{k=1}^{K}\left(\psi_{1}\left(\frac{n P+x_{0}}{k P+\alpha}\right)-\psi_{1}\left(\frac{n P+x_{0}}{k P+\beta}\right)\right) .
$$

As $n \rightarrow \infty$, the $k^{\text {th }}$-term in the sum tends to $\log \left(\frac{k P+\beta}{k P+\alpha}\right)=-\log \left(1-\frac{\beta-\alpha}{k P+\beta}\right) \geq \frac{\beta-\alpha}{k P+\beta}$. Thus

$$
\liminf _{n \rightarrow \infty} \int_{1-}^{n P+x_{0}} R^{\prime}\left(x_{0}\right)-R^{\prime}\left(P\left\{\frac{n P+x_{0}}{t P}\right\}\right) d \psi_{1}(t) \geq \delta(\beta-\alpha) \sum_{k=1}^{K} \frac{1}{k P+\beta} \geq \delta^{\prime} \log K
$$

for some $\delta^{\prime}>0$. This is true for every $K \geq 1$ so the lefthand side of (2.3) cannot be bounded. This contradiction proves the theorem.

Remark. (i) We see that $N(x)=c x+1-c$ determines an outer g-prime system for every $c>0$. What about g-prime systems; i.e. for which values of $c$ is $\pi$ increasing? We show in the appendix that this happens for $0<c \leq \lambda$ and fails for $c>\lambda$ for some $\lambda>2$.

(ii) The proof of Theorem 2.1 can be readily extended to the case where $R$ is absolutely continuous and $R^{\prime}(x)$ has a maximum value, say at $x=x_{0}$ and the set

$$
\left\{x \in[0, P]: R^{\prime}(x) \leq R^{\prime}\left(x_{0}\right)-\delta\right\}
$$

contains an interval, for some $\delta>0$.

In particular this shows that none of the functions $N_{\lambda}$ with $\lambda>1$ (as defined in [7], section 3) form part of a g-prime system, except of course when $\rho_{\lambda}=0$. (To recall: $N_{\lambda}(x)=x-R_{\lambda}(x)$ for $x \geq 1$ and zero otherwise, where $R_{\lambda}(x)$ is periodic with period 1 and defined for $0 \leq x<1$ by $R_{\lambda}(x)=$ $\rho_{\lambda}(\zeta(1-\lambda, 1-x)-\zeta(1-\lambda))$. Here $\rho_{\lambda}$ is a continuous function of $\lambda$ with $\rho_{1}=1$.)

For $\lambda>2$, this follows from Theorem 2.1 since $R_{\lambda}$ is continuously differentiable and non-constant. For $1<\lambda \leq 2$, this follows on noting that $R_{\lambda}$ is absolutely continuous and $R_{\lambda}^{\prime}$ is maximum at $0+$.

\section{§3. G-prime systems with $N(x)-c x$ periodic and finitely many discontinuities}

Suppose now that $N$ has discontinuities (other than at 1). To check whether $N$ comes from a g-prime system we consider its jump function $N_{J}$. By Theorem 1.1, a necessary condition that $N$ determines a g-prime system is that $N_{J}$ does.

Our strategy for determining the possible $N$ will be as follows. Writing $N=N_{J}+N_{C}$, we first show by extending Theorem 2.1 that we must have $N_{C}(x)=a(x-1)$ for some $a \geq 0$. Then we show that the discontinuities must occur at the (rational) integers and that the period, say $P$, is an integer. Writing $a_{n}$ for the jump at $n$ we therefore have $a_{n+P}=a_{n}$ for $n \geq 2$. Next we show that $a_{1+P}=a_{1}$ is forced, so $a_{n}$ is truly periodic. Using a result of Saias and Weingartner [8] on Dirichlet series with periodic coefficients, we deduce that (i) $a_{n}$ must be even $\left(\bmod P\right.$ ) and (ii) that $a_{n}$ is multiplicative. We are then in a position to deduce $N_{C} \equiv 0$ (i.e. $N$ is a step function) and determine exactly which arise from g-prime systems.

First we extend Theorem 2.1 to members of $T$.

\section{Theorem 3.1}

Let $N(x)=c x-R(x) \in T$, where $R$ is periodic and such that $\Pi$ is increasing. Then $N(x)=N_{J}(x)+$ $a(x-1)$ for some $a \geq 0$. 
Proof. We proceed as in the proof of Theorem 2.1 but with $R_{+}^{\prime}$ in place of $R^{\prime}$. Now (2.1) becomes

$$
N_{+}^{\prime}(x) \log x=\left(N_{+}^{\prime} * \psi_{1}\right)(x)+\left(N_{J, 1} * \psi_{+}^{\prime}\right)(x) \geq\left(N_{+}^{\prime} * \psi_{1}\right)(x),
$$

and (2.2) still holds with $R^{\prime}$ replaced by $R_{+}^{\prime}$. If $R_{+}^{\prime}$ is not constant, then as before, we can find an $x_{0} \in[0, P]$ which maximises $R_{+}^{\prime}$ and for which $R_{+}^{\prime}(x) \leq R_{+}^{\prime}\left(x_{0}\right)-\delta$ holds throughout some interval for some (sufficiently small) $\delta>0$. We obtain a contradiction as before and hence $N_{+}^{\prime}$ is constant.

But $N$ has finitely many discontinuities in bounded intervals, so $N_{+}^{\prime}=\left(N_{C}\right)_{+}^{\prime}$. So $N_{+}^{\prime} \equiv a$ implies (since $N_{C}$ is continuous) that $N_{C}(x)=a(x-1)$, using $N_{C}(1)=0$. Since $N_{C}$ is increasing, we must have $a \geq 0$.

Later on, we shall see that the only possible value of $a$ is 0 .

\section{Notation}

Let $\lambda$ denote the total jump of $N$ per interval of length $P$; i.e. $N_{J}(x+P)-N_{J}(x)=\lambda$ for $x \geq 1$. Thus $N_{J}(x)=\frac{\lambda}{P} x+O(1)$ and, by integration by parts, $\left(N_{J}\right)_{L}(x)=\frac{\lambda}{P} x \log x+O(x)$. Note that $\lambda=0$ implies $N$ is continuous, while $\lambda=c P$ implies $N=N_{J}$.

For the following, $D_{N}$ denotes the set of discontinuities of $N$ in $(0, \infty)$ and $D_{N}^{*}=D_{N} \cap(1, P+1]$. We suppose that $D_{N}^{*}$ is a finite, but non-empty, set.

\section{Proposition 3.2}

Let $D_{N}^{*}$ have $k$ elements. Suppose $\alpha \in D_{N}$ such that $\alpha$ is irrational. Then there are at most $k^{2}$ numbers $\beta \in D_{N}$ such that $\alpha \beta \in D_{N}$.

Proof. Suppose, for a contradiction, that there are $l>k^{2}$ numbers $\beta \in D_{N}$ such that $\alpha \beta \in D_{N}$. Let $D_{N}^{*}=\left\{c_{1}, \ldots, c_{k}\right\}$. Each $\beta$ is of the form $n P+c_{i}$. There are $k$ choices for $c_{i}$ so some $c_{i_{0}}$ will appear at least $k+1$ times. (If not and all appear at most $k$ times, then there can be at most $k^{2}$ such numbers $\beta$.)

Thus we have (at least) $k+1$ equations

$$
\alpha\left(n P+c_{i_{0}}\right)=m P+c_{j},
$$

with (possibly different) $m, n \in \mathbb{N}$ and some $c_{j} \in D_{N}^{*}$. As $D_{N}^{*}$ has only $k$ elements, at least one $c_{j}$ must occur twice; i.e. there exist positive integers $n_{1}, n_{2}, m_{1}, m_{2}$ such that

$$
\alpha\left(n_{1} P+c_{i_{0}}\right)=m_{1} P+c_{j_{0}} \quad \text { and } \quad \alpha\left(n_{2} P+c_{i_{0}}\right)=m_{2} P+c_{j_{0}} .
$$

Note that $n_{1} \neq n_{2}$ and $m_{1} \neq m_{2}$ otherwise they are not genuinely different equations. Subtracting these two gives

$$
\alpha\left(n_{2}-n_{1}\right)=m_{2}-m_{1},
$$

and $\alpha$ is rational - a contradiction.

\section{Proposition 3.3}

$D_{N}$ contains only rational numbers and $P$ is rational.

Proof. By 1.2(a) and Theorem 1.1,

$$
\left(N_{J}\right)_{L}(x)=\left(N_{J} * \psi_{J}\right)(x)=\sum_{\substack{\alpha \beta \leq x \\ \alpha, \beta \in D_{N}}} \Delta N(\alpha) \Delta \psi(\beta) .
$$

Since $\left(N_{J}\right)_{L}(x)=\frac{\lambda}{P} x \log x+O(x)$ and $D_{\psi} D_{N}=D_{N_{L}}=D_{N} \backslash\{1\}$, we may rewrite (3.1) as

$$
\sum_{\alpha \leq x} \Delta N(\alpha) \sum_{\substack{\beta \leq x / \alpha \\ \text { s.t. } \alpha \beta \in D_{N}}} \Delta \psi(\beta)=\frac{\lambda}{P} x \log x+O(x) .
$$


For $\alpha$ irrational, by Proposition 3.2 there are at most $k^{2}$ possible $\beta$ s for which $\alpha \beta \in D_{N}$, where $k=\left|D_{N}^{*}\right|$. For each such $\beta, \Delta \psi(\beta) \leq \Delta N(\beta) \log \beta \leq C \log \beta$ for some $C$. Hence the inner sum on the left of (3.2) is at most $C k^{2} \log (x / \alpha)$. Thus the contribution of irrational $\alpha$ to the LHS of (3.2) is less than

$$
C k^{2} \sum_{\alpha \leq x} \Delta N(\alpha) \log \frac{x}{\alpha}=C k^{2} \int_{1-}^{x} \log \frac{x}{t} d N_{J}(t)=C k^{2} \int_{1}^{x} \frac{N_{J}(t)}{t} d t=O(x) .
$$

Hence

$$
\sum_{\substack{\alpha \leq x \\ \alpha \text { rational }}} \Delta N(\alpha) \sum_{\substack{\beta \leq x / \alpha \\ \text { s.t. } \alpha \beta \in D_{N}}} \Delta \psi(\beta)=\frac{\lambda}{P} x \log x+O(x) .
$$

But the LHS of (3.3) is (using Beurling's PNT for $\psi_{J}(x)$ )

$$
\sum_{\substack{\alpha \leq x \\ \alpha \text { rational }}} \Delta N(\alpha) \psi_{J}\left(\frac{x}{\alpha}\right) \sim x \sum_{\substack{\alpha \leq x \\ \alpha \text { rational }}} \frac{\Delta N(\alpha)}{\alpha} .
$$

Now the function

$$
N_{J, \mathbb{Q}}(x) \stackrel{\text { def }}{=} \sum_{\substack{\alpha \leq x \\ \alpha \text { rational }}} \Delta N(\alpha)=\frac{\mu}{P} x+O(1)
$$

for some $\mu \leq \lambda$ by periodicity. ( $\mu$ is the jump per interval of length $P$ from the rational discontinuities.) The RHS of (3.4) is therefore

$$
x \int_{1}^{x} \frac{1}{t} d N_{J, \mathbb{Q}}(t)=x \int_{1}^{x} \frac{N_{J, \mathbb{Q}}(t)}{t^{2}} d t+O(x)=\frac{\mu}{P} x \log x+O(x) .
$$

It follows that $\mu=\lambda$ and there are no irrational numbers in $D_{N}$.

Finally, $\alpha \in D_{N}$ with $\alpha>1$ implies $\alpha+P \in D_{N}$ by periodicity. As $D_{N}$ contains only rationals, this forces $P$ rational.

\section{Proposition 3.4}

$D_{N} \subset \mathbb{N}$ and $P \in \mathbb{N}$.

Proof. Since $D_{N} \backslash\{1\}=D_{\psi * N}=D_{\psi} D_{N}$, if $\alpha \in D_{\psi}$ then $\alpha \beta \in D_{N}$ for every $\beta \in D_{N}$. In particular (using $\left.D_{\psi} \subset D_{N}\right) \alpha \in D_{\psi}$ implies $\alpha^{n} \in D_{N}$ for every $n \in \mathbb{N}$. By periodicity, $\alpha^{n}-k P \in D_{N}$ for every integer $k$ provided $\alpha^{n}-k P \geq 1$.

Now write $\alpha=r / s$ and $P=t / u$ where $r, s, t, u \in \mathbb{N}$ and $(r, s)=(t, u)=1$. For $D_{N}^{*}$ to be finite, the numbers $1+P\left\{\frac{\alpha^{n}-1}{P}\right\}(n=1,2,3 \ldots)$ (take $k=\left[\frac{\alpha^{n}-1}{P}\right]$ above) must repeat themselves infinitely often; i.e. for infinitely many values of $n$,

$$
\alpha^{n}-k P=\alpha^{n_{0}}-k_{0} P
$$

for some integers $k, k_{0}$, and $n_{0}$. As such,

$$
P=\frac{\alpha^{n}-\alpha^{n_{0}}}{k-k_{0}}=\frac{\left(\frac{r}{s}\right)^{n}-\left(\frac{r}{s}\right)^{n_{0}}}{k-k_{0}}=\frac{t}{u} .
$$

Multiplying through by $\left(k-k_{0}\right) u s^{n_{0}}$ shows that $s^{n-n_{0}} \mid u r^{n}$ for infinitely many $n$. But $(r, s)=1$, so $s^{n-n_{0}} \mid u$ for infinitely many $n$. This is only possible is $s=1$; i.e. $\alpha \in \mathbb{N}$. Hence $D_{\psi} \subset \mathbb{N}$.

It follows that $D_{\Pi} \subset \mathbb{N}$ also, and $D_{\Pi^{* k}} \subset \mathbb{N}$ for every positive integer $k$. Since $N=\sum_{k=0}^{\infty} \Pi^{* k} / k$ ! , it follows that $D_{N} \subset \mathbb{N}$ also.

Finally, $m \in D_{N}$ with $m>1$ implies $m+P \in D_{N}$ by periodicity. Since $D_{N} \subset \mathbb{N}$, this implies $P \in \mathbb{N}$. 


\section{$\S 4$. Determining the jumps}

Now that we have established the discontinuities are at the integers, it remains to determine the possible jumps. Write $a_{n}=\Delta N(n)$ and $c_{n}=\Delta \psi(n)$. Thus $a_{1}=1$ and $a_{n+P}=a_{n}$ for $n>1$. The equation $\Delta N_{L}=(\Delta N) * \psi_{J}$ translates as

$$
a_{n} \log n=\sum_{d \mid n} c_{d} a_{n / d}
$$

Thus $c_{1}=0$, for a prime $p, c_{p}=a_{p} \log p$, while for distinct primes $p$ and $q$, we have (after some calculation) $c_{p q}=\left(a_{p q}-a_{p} a_{q}\right) \log p q$.

Next we show that $a_{n}$ is truly periodic $\left(a_{n+P}=a_{n}\right.$ for $\left.n \geq 1\right)$. For the proof, let $\left\langle\mathbb{P}_{r, P}\right\rangle$ denote the set of numbers of the form $p_{1} \ldots p_{k}$ where the $p_{i}$ are distinct primes, all congruent to $r(\bmod P)$. Here $r$ is coprime to $P$. Each such set is infinite by Dirichlet's Theorem on primes in arithmetic progressions.

\section{Proposition 4.1}

$a_{P+1}=1$.

Proof. First we prove that $a_{P+1}=0$ or 1.

Let $p_{1}, \ldots, p_{k}$ be distinct primes all of the form $1(\bmod P)$, with $k \geq 3$. Let $n=p_{1} \ldots p_{k}$, which is also $1(\bmod P)$. Note that for every $d \mid n, d=1(\bmod P)$, so that $a_{d}=a_{P+1}$ if $d>1$. In particular $c_{p_{i} p_{j}}=a_{P+1}\left(1-a_{P+1}\right) \log p_{i} p_{j}$ for any $1 \leq i, j \leq k$ with $i \neq j$. Since $c_{n} \geq 0$, (4.1) implies

$$
a_{P+1} \log n \geq \sum_{1 \leq i<j \leq k} c_{p_{i} p_{j}} a_{n / p_{i} p_{j}}=a_{P+1}^{2}\left(1-a_{P+1}\right) \sum_{1 \leq i<j \leq k} \log p_{i} p_{j}=a_{P+1}^{2}\left(1-a_{P+1}\right)(k-1) \log n .
$$

This is impossible for $k$ sufficiently large unless $a_{P+1}$ equals 0 or 1.

Next we show that $a_{P+1}=0$ implies $a_{n}=0$ for all $n>1$, and hence that $N_{J}(x)=1$ for $x \geq 1-$ i.e. the continuous case.

By induction. Suppose $a_{P+1}=0$ and that $a_{n}=0$ for all $n>1$ such that ${ }^{5} \Omega(n)<k$, some $k \geq 1$. (It is vacuously true for $k=1$.) Then $a_{n r}=0$ for all such $n$ and all $r \equiv 1(\bmod P)$, by periodicity. In particular, if we take $r \in\left\langle\mathbb{P}_{1, P}\right\rangle$. Note that this implies $c_{n r}=0$ also for such $n$ and $r$.

Now let $n$ be such that $\Omega(n)=k$. Then, with $r \in\left\langle\mathbb{P}_{1, P}\right\rangle$ such that $(n, r)=1$,

$$
a_{n r} \log n r=\sum_{d \mid n r} c_{d} a_{n r / d}=\sum_{d_{1} \mid n} \sum_{d_{2} \mid r} c_{d_{1} d_{2}} a_{n r / d_{1} d_{2}} .
$$

Now $d_{2} \in\left\langle\mathbb{P}_{1, P}\right\rangle$ also, so by assumption, $c_{d_{1} d_{2}}=0$ if $\Omega\left(d_{1}\right)<k$. Hence only the terms with $\Omega\left(d_{1}\right)=k$ give a contribution; i.e. only if $d_{1}=n$. Also $a_{n r}=a_{n}$ by periodicity. Thus

$$
a_{n} \log n r=\sum_{d_{2} \mid r} c_{n d_{2}} a_{r / d_{2}}=c_{n r},
$$

since only the term with $d_{2}=r$ makes $a_{r / d_{2}}$ non-zero.

Now consider $a_{n^{2} r}$ with $n$ and $r$ as above. We have

$$
a_{n^{2} r} \log n^{2} r \geq \sum_{d \mid r} c_{n d} a_{n r / d}
$$

Using (4.2) and noting that $a_{n^{2} r}=a_{n^{2}}$, we therefore have ${ }^{6}$

$$
a_{n^{2}} \log n^{2} r \geq a_{n}^{2} \sum_{d \mid r} \log n d=\frac{a_{n}^{2}}{2} d(r) \log n^{2} r .
$$

\footnotetext{
${ }^{5}$ As usual, $\Omega(n)$ denotes the total number of prime factors of $n ; \omega(n)$ denotes the number of distinct prime factors of $n$.

${ }^{6}$ Using $2 \sum_{d \mid n} \log k d=d(n) \log k^{2} n$.
} 
i.e. $2 a_{n^{2}} \geq a_{n}^{2} d(r)$ for all $r \in\left\langle\mathbb{P}_{1, P}\right\rangle$ such that $(n, r)=1$. But $r$ can be chosen such that $d(r)$ is arbitrarily large, and we have a contradiction if $a_{n}>0$. Thus $a_{n}=0$ is forced.

Hence by induction, $a_{n}=0$ for all $n>1$.

Thus, for the discontinuous case, $\hat{N}_{J}(s)$ is a Dirichlet series with purely periodic coefficients. Further, if $N_{J}$ determines a g-prime system, then $\hat{N}_{J}$ has no zeros in ${ }^{7} H_{1}$. Now we use the main result of Saias and Weingartner ([8], Corollary): Let $F$ be a Dirichlet series with periodic coefficients. Then $F$ does not vanish in $H_{1}$ if and only if $F=P L_{\chi}$, where $P$ is a Dirichlet polynomial with no zeros in $H_{1}$ and $\chi$ is a Dirichlet charcter.

Thus $\hat{N}_{J}=P L_{\chi}$ for some Dirichlet polynomial $P$ and Dirichlet character $\chi$. We shall see below that the positivity of the coefficients of $\hat{N}_{J}$ implies that $\chi$ must be a principal character, showing that we actually have $\hat{N}_{J}=Q \zeta$ for some Dirichlet polynomial $Q$.

\section{Proposition 4.2}

$\hat{N}_{J}(s)=Q(s) \zeta(s)$ where $Q$ is a Dirichlet polynomial with no zeros in $H_{1}$. Furthermore, $a_{n}$ is even (mod $P)$; i.e. $a_{n}=a_{(n, P)}$, and $Q(s)=\sum_{d \mid P} \frac{q(d)}{d^{s}}$ for some $q(d)$.

Proof. From above, $\hat{N}_{J}(s)=P(s) L_{\chi}(s)$, where $P(s)=\sum_{n=1}^{N} b_{n} n^{-s}$ say. Extend $b_{n}$ so that $b_{n}=0$ for $n>N$. By inversion,

$$
b_{n}=\sum_{d \mid n} \mu(d) \chi(d) a_{n / d}=0 \quad \text { for } n>N .
$$

In particular, for every prime $p>N, a_{p}=\chi(p)$. A simple induction on $\Omega(n)$ shows that more generally, $a_{n}=\chi(n)$ whenever all the prime factors of $n$ are greater than $N$. Consequently, for all such $n, a_{n}=0$ or 1 (since $a_{n} \geq 0$ while $\chi(n)=0$ or a root of unity).

Now let $p>\max \{N, P\}$ be prime. Then $p \equiv r(\bmod P)$ for some $r$ with $(r, P)=1$. Let $n=p^{\phi(P)}$. Then $n \equiv r^{\phi(P)} \equiv 1(\bmod P)$ and hence

$$
1=a_{1}=a_{n}=\chi(n)=\chi\left(p^{\phi(P)}\right)=\chi(p)^{\phi(P)} .
$$

But $\chi(p)=0$ or 1 , so $\chi(p)=1$ for all sufficiently large $p$.

This implies $\chi$ must be a principal character. For suppose $\chi$ is a character modulo $m$. Let $(r, m)=1$. For a sufficiently large prime $p$ in each residue class $r(\bmod m), 1=\chi(p)=\chi(r)$ by periodicity. Thus $\chi(r)=1$ whenever $(r, m)=1$; i.e. $\chi$ is principal. Thus

$$
\hat{N}_{J}(s)=P(s) L_{\chi_{0}}(s)=P(s) \zeta(s) \prod_{p \mid m}\left(1-\frac{1}{p^{s}}\right)=Q(s) \zeta(s),
$$

where $Q$ is again a Dirichlet polynomial, non-zero in $H_{1}$. Denoting the coefficients of $Q$ by $q(n)$, we see that $q(1)=1, q(n)=0$ for $n$ sufficiently large, and

$$
a_{n}=\sum_{d \mid n} q(d) .
$$

To show $a_{n}$ is even $(\bmod P)$, we first show that for $d \mid P, a_{p d}=a_{d}$ for all sufficiently large primes $p$. It is true for $d=1$, so suppose it is true if $\Omega(d)<k$, for some $k \geq 1$. Then

Let $d \mid P$ such that $\Omega(d)=k$. Let $p$ be prime and sufficiently large so that $(p, d)=1$ and $q(p d)=0$.

$$
\begin{aligned}
0 & =q(p d)=\sum_{c \mid p d} \mu(c) a_{p d / c}=\sum_{c \mid d} \mu(c) a_{p d / c}+\sum_{c \mid d} \mu(p c) a_{d / c} \\
& =a_{p d}+\sum_{\substack{c \mid d \\
c>1}} \mu(c) a_{p d / c}-\sum_{c \mid d} \mu(c) a_{d / c}=a_{p d}-a_{d}
\end{aligned}
$$

${ }^{7}$ For $\theta \in \mathbb{R}, H_{\theta}$ denotes the half-plane $\{s \in \mathbb{C}: \Re s>\theta\}$. 
since $a_{p d / c}=a_{d / c}$ as $\Omega(d / c)<k$ in the first sum.

Let $d=(n, P)$. Then $\left(\frac{n}{d}, \frac{P}{d}\right)=1$ and there exist arbitrarily large primes $p$ congruent to $\frac{n}{d}\left(\bmod \frac{P}{d}\right)$. For such primes $p, p d \equiv n(\bmod P)$, and by periodicity $a_{n}=a_{p d}=a_{d}$ for $p$ sufficiently large. Thus $a_{n}=a_{(n, P)}$.

As a result, we can write

$$
\hat{N}_{J}(s)=\sum_{d \mid P} \sum_{\substack{n=1 \\(n, P)=d}}^{\infty} \frac{a_{n}}{n^{s}}=\sum_{d \mid P} \frac{a_{d}}{d^{s}} \sum_{\substack{m=1 \\(m, P / d)=1}}^{\infty} \frac{1}{m^{s}}=\sum_{d \mid P} \frac{a_{d}}{d^{s}} \prod_{p \mid P / d}\left(1-\frac{1}{p^{s}}\right) \zeta(s)=Q(s) \zeta(s),
$$

which shows that $q(n)$ is supported on the divisors of $P$.

Next we show that $a_{n}$ is multiplicative.

\section{Theorem 4.3}

$a_{n}$ is multiplicative.

Proof. Equivalently, we show $q(n)$ is multiplicative. Let the period be $P=p_{1}^{m_{1}} \ldots p_{k}^{m_{k}}$. Write

$$
Q(s)=\sum_{d \mid P} \frac{q(d)}{d^{s}}=\exp \left\{\sum_{n=1}^{\infty} \frac{t(n)}{n^{s}}\right\},
$$

for some $t(n)$, where $t(1)=0$. Since $\hat{N}_{J}(s)=\exp \left\{\sum_{n=1}^{\infty} \frac{b_{n}}{n^{s}}\right\}$ for some $b_{n} \geq 0$, Proposition 4.2 implies that $t(n)=b_{n} \geq 0$ for $n$ not a prime power. The aim is to show that $t(n)=0$ for such $n$.

Since the $q(n)$ are supported on the divisors of $P, t(n)$ is supported on the set $\left\{p_{1}^{n_{1}} \ldots p_{k}^{n_{k}}: n_{1}, \ldots, n_{k} \in\right.$ $\left.\mathbb{N}_{0}\right\}$.

For each $p \mid P$ let

$$
Q_{p}(s)=\sum_{r=0}^{\infty} \frac{q\left(p^{r}\right)}{p^{r s}}
$$

(This is a polynomial in $p^{-s}$.) Then

$$
\prod_{p \mid P} Q_{p}(s)=\exp \left\{\sum_{n \text { prime power }} \frac{t(n)}{n^{s}}\right\}
$$

where the sum is over prime powers only. Now define $T_{1}(s)$ and $t_{1}(n)$ by

$$
\frac{Q(s)}{\prod_{p \mid P} Q_{p}(s)}=\exp \left\{T_{1}(s)\right\}=\exp \left\{\sum_{n=1}^{\infty} \frac{t_{1}(n)}{n^{s}}\right\}
$$

i.e. $t_{1}(n)=t(n)$ for $n$ not a prime power and zero otherwise.

If the Dirichlet series for $T_{1}(s)$ converges everywhere, then the result follows. For the LHS of (4.3) is then entire and of order 1 while if $t_{1}\left(n_{0}\right)>0$ for some $n_{0}>1$, then the RHS of $(4.3)$ is, for negative $s$, at least $e^{t_{1}\left(n_{0}\right) n_{0}^{-s}}$, which has infinite order. The contradiction implies $T_{1}$ is identically zero and $Q=\prod_{p} Q_{p}$.

Suppose then that the series for $T_{1}$ has a finite abscissa of convergence, say $-\beta$. Since the coefficients are non-negative, $-\beta$ must be a singularity of the function; i.e. $-\beta$ must be a zero of one of the $Q_{p}(s)$. (As we shall see later, $Q_{p}(s) \neq 0$ in $H_{0}$, so $\beta \geq 0$, but we do not require to know this at this stage.)

We can write down the 'spatial extension' of (4.3). We can think of this as substituting $z_{i}=p_{i}^{-s}$. For $p$ prime, let $\tilde{Q}_{p}(z)=\sum_{r=0}^{\infty} q\left(p^{r}\right) z^{r}$, so that $\tilde{Q}_{p}\left(p^{-s}\right)=Q_{p}(s)$. Now define

$$
\tilde{Q}\left(z_{1}, \ldots, z_{k}\right)=\sum_{b_{1}, \ldots, b_{k} \geq 0} q\left(p_{1}^{b_{1}} \ldots p_{k}^{b_{k}}\right) z_{1}^{b_{1}} \ldots z_{k}^{b_{k}},
$$


(the series is of course finite) and similarly for $\tilde{T}_{1}$. Then (4.3) becomes

$$
\frac{\tilde{Q}\left(z_{1}, \ldots, z_{k}\right)}{\tilde{Q_{p_{1}}}\left(z_{1}\right) \ldots \tilde{Q_{p_{k}}}\left(z_{k}\right)}=\exp \left\{\tilde{T}_{1}\left(z_{1}, \ldots, z_{k}\right)\right\}=\exp \left\{\sum_{n_{1}, \ldots, n_{k} \geq 0} t_{1}\left(p_{1}^{n_{1}} \ldots p_{k}^{n_{k}}\right) z_{1}^{n_{1}} \ldots z_{k}^{n_{k}}\right\}
$$

Since (4.3) holds for $\sigma>-\beta$, (4.4) holds in the domain $\left\{\left(z_{1}, \ldots, z_{k}\right):\left|z_{1}\right|<p_{1}^{\beta}, \ldots,\left|z_{k}\right|<p_{k}^{\beta}\right\}$.

Let $r$ be the smallest positive integer such that $t_{1}(n)=0$ whenever $\omega(n)<r$. (Thus $2 \leq r \leq k$ ). Put $z_{r+1}, \ldots, z_{k}=0$. Then (4.4) becomes

$$
\frac{\tilde{Q}\left(z_{1}, \ldots, z_{r}\right)}{\tilde{Q_{p_{1}}}\left(z_{1}\right) \ldots \tilde{Q_{p_{r}}}\left(z_{r}\right)}=\exp \left\{\sum_{n_{1}, \ldots, n_{r} \geq 0} t_{1}\left(p_{1}^{n_{1}} \ldots p_{r}^{n_{r}}\right) z_{1}^{n_{1}} \ldots z_{r}^{n_{r}}\right\}
$$

where we identified $\tilde{Q}\left(z_{1}, \ldots, z_{r}\right)$ with $\tilde{Q}\left(z_{1}, \ldots, z_{r}, 0, \ldots, 0\right)$. Without loss of generality, we may assume that the numerator and denominator of the left-hand side of (4.5) have no common factors. (If there are any, cancel them, and apply the argument to what remains.)

Let $z_{i}=x_{i}(i=1, \ldots, r)$ be real and positive. Take logs of $(4.5)$ and differentiate with respect to each of the variables $x_{1}, \ldots, x_{r}$. This gives

$$
\sum_{n_{1}, \ldots, n_{r} \geq 0} n_{1} \ldots n_{r} t_{1}\left(p_{1}^{n_{1}} \ldots p_{r}^{n_{r}}\right) x_{1}^{n_{1}} \ldots x_{r}^{n_{r}}=\frac{\partial^{r}}{\partial x_{1} \ldots \partial x_{r}} \log \tilde{Q}\left(x_{1}, \ldots, x_{r}\right)=\frac{P\left(x_{1}, \ldots, x_{r}\right)}{\tilde{Q}\left(x_{1}, \ldots, x_{r}\right)^{r}}
$$

for some polynomial $P$. The crucial point here is that the polynomials $\tilde{Q}_{p}$ have all disappeared.

Now, $\tilde{Q}_{p}\left(p^{\beta}\right)=0$ for some $p \mid P$, say $p=p_{1}$. Fix $x_{2}, \ldots, x_{r}$ and let $x_{1} \rightarrow p_{1}^{\beta}$ through real values from below. If $\tilde{Q}\left(p_{1}^{\beta}, x_{2}, \ldots, x_{r}\right) \neq 0$, then the RHS of (4.6) remains bounded, and hence (since $\left.t_{1}(n) \geq 0\right)$, the series

$$
\sum_{n_{1}, \ldots, n_{k} \geq 1} n_{1} \ldots n_{r} t_{1}\left(p_{1}^{n_{1}} \ldots p_{r}^{n_{r}}\right) p_{1}^{n_{1} \beta} x_{2}^{n_{2}} \ldots x_{r}^{n_{r}} \quad \text { converges }
$$

while the LHS of (4.5) tends to infinity, so

$$
\sum_{n_{1}, \ldots, n_{r} \geq 0} t_{1}\left(p_{1}^{n_{1}} \ldots p_{r}^{n_{r}}\right) p_{1}^{n_{1} \beta} x_{2}^{n_{2}} \ldots x_{r}^{n_{r}} \quad \text { diverges. }
$$

But (4.7) and (4.8) are in contradiction since in (4.8) we actually require $n_{1}, \ldots, n_{r} \geq 1$ (if any $n_{j}=0$, there is no contribution to the sum as $\left.\omega\left(p_{1}^{n_{1}} \ldots p_{r}^{n_{r}}\right)<r\right)$.

Thus this forces $\tilde{Q}\left(p_{1}^{\beta}, x_{2}, \ldots, x_{r}\right)=0$ for every $x_{i}(i=2, \ldots, r)$ in some interval, and hence for all such $x_{i}$, since $\tilde{Q}$ is a polynomial. But this implies $\left(x_{1}-p_{1}^{\beta}\right)$ is a factor of both $\tilde{Q}\left(x_{1}, \ldots, x_{r}\right)$ and $\tilde{Q_{p_{1}}}\left(x_{1}\right)$ - a contradiction. Hence $T_{1}$ is identically zero and the result follows.

Determining $a$ for which $N_{J}(x)+a(x-1)$ is a g-prime system

The problem thus reduces to determining $Q_{p}(s)$. We shall see in Theorem 4.4 that the zeros of $Q_{p}(s)$ all have real part less than or equal to zero. We use this fact to deduce that the only permissible value of $a$ is 0 .

For, using this fact, the zeros of $Q$ then all lie in $\mathbb{C} \backslash H_{0}$. In particular, in $H_{0}$, the zeros of $\hat{N}_{J}$ are precisely the zeros of $\zeta$ and hence $\hat{N}_{J}$ has no real positive zeros. Indeed, $Q(\sigma)>0$ for $\sigma>0$ since $Q(\sigma)$ is real and non-zero here and as $\sigma \rightarrow \infty, Q(\sigma) \rightarrow 1$. Thus $\hat{N}_{J}(\sigma)<0$ for $0<\sigma<1$. Also $\hat{N}(\sigma)=\hat{N}_{J}(\sigma)-\frac{a}{1-\sigma}<0$ for $\sigma \in(0,1)$.

Now $N=N_{J}+N_{C}$ and $\psi=\psi_{J}+\psi_{C}$ and by assumption $\psi_{C}$ is increasing. (Here $N_{C}(x)=a(x-1)$, so that $\hat{N}_{C}(s)=\frac{a}{s-1}$.) Thus

$$
\hat{\psi_{C}}(s)=\hat{\psi}(s)-\hat{\psi}_{J}(s)=\frac{\hat{N}_{J}^{\prime}(s)}{\hat{N}_{J}(s)}-\frac{\hat{N}^{\prime}(s)}{\hat{N}(s)},
$$


since $\left(\Pi_{J}, N_{J}\right)$ and $(\Pi, N)$ are g-prime systems. Note that $\hat{\psi_{C}} \neq-{\hat{N_{C}}}^{\prime} /{\hat{N_{C}}}_{C}$ as $\left(\Pi_{C}, N_{C}\right)$ is not a g-prime system (indeed $N_{C}(1)=0$ ).

Both $\psi(s)$ and $\psi_{J}(s)$ are meromorphic functions, holomorphic in $\overline{H_{1}} \backslash\{1\}$, with simple poles at $s=1$ and residue 1 . Thus $\psi_{C}(s)$ has a removable singularity at 1 and poles at the zeros of $\hat{N}$ and $\hat{N}_{J}$.

Landau's Oscillation Theorem (cf. [3], p.137) applied to $\hat{\psi_{C}}$ implies that $\hat{\psi_{C}}$ has a singularity at its abscissa of convergence, say $\theta$. Of course $\theta<1$ must be a zero of $\hat{N}$ or $\hat{N}_{J}$. But neither $\hat{N}$ nor $\hat{N}_{J}$ has real positive zeros, so $\theta \leq 0$. But then $\hat{\psi_{C}}$ must be holomorphic in $H_{0}$, implying that $\hat{N}$ and $\hat{N}_{J}$ have the same zeros here; i.e. all the non-trivial Riemann zeros. But at each such zero, say $\rho, \hat{N}_{C}(\rho)=0$ also. This is impossible as $\hat{N}_{C}$ has no zeros, except if $a=0$.

Hence $a=0$ is forced and $N=N_{J}$.

\section{Criteria for g-primes}

We have $\hat{N}(s)=Q(s) \zeta(s)=\exp \{T(s)+\log \zeta(s)\}=\exp \{\hat{\Pi}(s)\}$. Thus

$$
\hat{\Pi}(s)=\sum_{n=1}^{\infty} \frac{\Lambda_{1}(n)+t(n)}{n^{s}} .
$$

For $\Pi$ to be increasing, the coefficients of $\hat{\Pi}$ must be non-negative; i.e. $\Lambda_{1}(n)+t(n) \geq 0$ for all $n \in \mathbb{N}$. As $t(n)$ is supported on the powers of the prime divisors of $P$, we have:

$$
\Pi \text { is increasing } \Longleftrightarrow t\left(p^{k}\right) \geq-\frac{1}{k} \quad \text { for } p \mid P \text { and } k \in \mathbb{N} .
$$

Note that $t(p)=q(p)=a_{p}-1 \geq-1$ for $p$ prime, so $(*)$ is satisfied for $k=1$.

Turning now to $\pi(x), N$ determines g-primes if $\pi$ is increasing where $\pi(x)=\sum_{k=1}^{\infty} \frac{\mu(k)}{k} \Pi\left(x^{1 / k}\right)$. But

$$
\hat{\pi}(s)=\sum_{k=1}^{\infty} \frac{\mu(k)}{k} \hat{\Pi}(k s)=\sum_{p} \frac{1}{p^{s}}+\sum_{k, n \geq 1} \frac{\mu(k) t(n)}{k n^{k s}}=\sum_{n=1}^{\infty} \frac{\pi_{n}}{n^{s}},
$$

say, for some coefficients $\pi_{n}$. Thus $\pi$ is increasing if and only if $\pi_{n} \geq 0$ for all $n$. Now $\pi_{1}=0$ and $\pi_{p}=1+t(p) \geq 0$ for $p$ prime, while $\pi_{n}=0$ for $n$ not a prime power. Hence

$$
\pi \text { is increasing } \Longleftrightarrow \sum_{d \mid n} \frac{\mu(d)}{d} t\left(p^{n / d}\right) \geq 0 \quad \text { for } n \geq 2 \text { and } p \mid P \text {. }
$$

To deal with these criteria, it is useful to write them in terms of the zeros of $\tilde{Q}_{p}$.

The zeros of $\tilde{Q}_{p}$

Let $p \mid P$ and let $k$ be the degree of $\tilde{Q}_{p}$. Then $\tilde{Q_{p}}$ has $k$ zeros $\lambda_{1}, \ldots, \lambda_{k}$. Letting $\mu_{r}=1 / \lambda_{r}$ gives $\tilde{Q}_{p}(z)=\left(1-\mu_{1} z\right) \ldots\left(1-\mu_{k} z\right)$ and

$$
\log \tilde{Q}_{p}(z)=\sum_{r=1}^{k} \log \left(1-\mu_{r} z\right)=-\sum_{n=1}^{\infty}\left(\frac{1}{n} \sum_{r=1}^{k} \mu_{r}^{n}\right) z^{n} .
$$

Since $\log \tilde{Q}_{p}(z)=\sum_{r=1}^{\infty} t\left(p^{r}\right) z^{r}$, equating coefficients gives

$$
t\left(p^{n}\right)=-\frac{1}{n} \sum_{r=1}^{k} \mu_{r}^{n}
$$

Hence $(*)$ is satisfied for a prime $p \mid P$ if and only if

$$
\tau_{n}:=\sum_{r=1}^{k} \mu_{r}^{n} \leq 1 \quad \text { for } n \in \mathbb{N} .
$$


Turning to $(* *)$, let $s_{n}(w)=\sum_{d \mid n} \mu(d) w^{n / d}$ for $w \in \mathbb{C}$. Then $\sum_{d \mid n} \frac{\mu(d)}{d} t\left(p^{n / d}\right)=-\frac{1}{n} \sum_{r=1}^{k} s_{n}\left(\mu_{r}\right)$ and $(* *)$ is satisfied for a prime $p \mid P$ if and only if

$$
\sum_{r=1}^{k} s_{n}\left(\mu_{r}\right) \leq 0 \quad \text { for } n \geq 2
$$

\section{Theorem 4.4}

Let $\tilde{Q}_{p}, k$ and $\mu_{1}, \ldots, \mu_{k}$ be as above. For $k=1$, (†) is satisfied if and only if $\left|\mu_{1}\right| \leq 1$. For $k>1$, if ( $\dagger$ ) is satisfied, then $\left|\mu_{r}\right|<1$ for all $r$.

Proof. For $k=1$ this is trivial so assume $k>1$ and that $(\dagger)$ is satisfied. The numbers $\mu_{1} \ldots, \mu_{k}$ are either real or occur in complex conjugate pairs. Denote the real ones by $\mu_{1}, \ldots, \mu_{l}$ and the complex ones by $\nu_{1} e^{ \pm i \theta_{1}}, \ldots, \nu_{m} e^{ \pm i \theta_{m}}$ where $\nu_{r}>0$ and $0<\theta_{r}<\pi$. Thus ( $\left.\dagger\right)$ becomes

$$
\tau_{n}=\mu_{1}^{n}+\ldots+\mu_{l}^{n}+2\left(\nu_{1}^{n} \cos n \theta_{1}+\ldots \nu_{m}^{n} \cos n \theta_{m}\right) \leq 1 .
$$

Assume without loss of generality that $\left|\mu_{1}\right| \geq \ldots \geq\left|\mu_{l}\right|$ and $\nu_{1} \geq \ldots \geq \nu_{m}$. If $\left|\mu_{1}\right| \geq 1$, then $\mu_{1}^{2 n} \geq 1$ and (4.9) implies

$$
\nu_{1}^{2 n} \cos 2 n \theta_{1}+\ldots+\nu_{m}^{2 n} \cos 2 n \theta_{m} \leq 0 \quad \text { for all } n \in \mathbb{N} .
$$

Suppose $\nu_{1}=\ldots=\nu_{q}>\nu_{q+1}$ for some $q \leq m$, then this involves

$$
\cos 2 n \theta_{1}+\ldots+\cos 2 n \theta_{q} \leq \frac{a}{A^{n}} \quad(n \in \mathbb{N})
$$

for some $a$ and $A>1$. But this is impossible as we show below.

Thus if any $\mu_{r}$ is real, then $\left|\mu_{r}\right|<1$. Now suppose $\nu_{1}=\ldots=\nu_{q}>\nu_{q+1}$ and $\nu_{1} \geq 1$. Then (4.9) implies

$$
\cos 2 n \theta_{1}+\ldots+\cos 2 n \theta_{q} \leq \frac{1}{2}+\frac{a}{A^{n}} \quad(n \in \mathbb{N})
$$

for some $a$ and $A>1$. We show this is impossible, which in turn implies (4.10) is impossible.

Let $\phi_{r}=\theta_{r} / \pi$. By Dirichlet's Theorem (see [6], p.170), the numbers $n \phi_{1}, \ldots, n \phi_{q}$ can be made arbitrarily close to $q$ integers simultaneously; i.e. given $\varepsilon>0$, there exists $n \in \mathbb{N}$ such that $\left|n \phi_{r}-K_{r}\right|<\varepsilon$ for $r=1, \ldots, q$ and integers $K_{r}$. Thus, for some $\left|\delta_{r}\right|<\varepsilon$

$$
\cos 2 n \theta_{r}=\cos 2 \pi n \phi_{r}=\cos 2 \pi\left(K_{r}+\delta_{r}\right)=\cos 2 \pi \delta_{r}>\cos 2 \pi \varepsilon,
$$

which can be made as close to 1 as we please. The inequalities (4.10) and (4.11) are impossible and hence $\nu_{r}<1$ for all $r$.

To deal with $(\dagger \dagger)$ we require the following.

\section{Lemma 4.5}

(a) Let $w \in \mathbb{R}$. Then $s_{n}(w) \leq 0$ for all $n>1$ if and only if $w=0$ or 1 .

(b) Let $w_{1}, \ldots, w_{k}$ be non-zero complex numbers of modulus less than one, and symmetric about $\mathbb{R}$; i.e. $\overline{w_{i}}=w_{j}$ for some $j$. Then $s_{n}\left(w_{1}\right)+\cdots+s_{n}\left(w_{k}\right)$ changes sign infinitely often.

Proof. (a) For $p$ prime, $s_{p}(w)=w^{p}-w>0$ for $w>1$, while for $p$ an odd prime, $s_{2 p}(w)=w^{2 p}-w^{p}-$ $w^{2}+w>0$ whenever $w<-1$ for $p$ sufficiently large. This leaves $-1 \leq w \leq 1$. For $w=1, s_{n}(w)=0$ for $n>1$, while for $w=-1, s_{n}(w)=0$ for $n>2$ and $s_{2}(-1)=2$, so it narrowly fails in this case. For $w=0$ the result holds trivially.

Now suppose $-1<w<1, w \neq 0$. Consider the entire function defined by the Dirichlet series

$$
H_{w}(s)=\sum_{n=1}^{\infty} \frac{w^{n}}{n^{s}} .
$$


Note that

$$
\frac{H_{w}(s)}{\zeta(s)}=\sum_{n=1}^{\infty} \frac{s_{w}(n)}{n^{s}}
$$

Now if $s_{n}(w)$ is ultimately of one sign, then the abscissa of convergence of this series must be a singularity of $H_{w} / \zeta$. This singularity must be real, and there can be no others further to the right. But the first real singularity (furthest to the right) is at -2 , so $H_{w}$ must be zero at all the complex zeros of $\zeta$. This is a contradiction as $H_{w}$, being bounded in any strip, has at most $O(T)$ zeros up to height $T$ here.

(b) This time

$$
\frac{H_{w_{1}}(s)+\cdots+H_{w_{k}}(s)}{\zeta(s)}=\sum_{n=1}^{\infty} \frac{s_{n}\left(w_{1}\right)+\cdots+s_{n}\left(w_{k}\right)}{n^{s}} .
$$

If $s_{n}\left(w_{1}\right)+\cdots+s_{n}\left(w_{k}\right)$ is ultimately of one sign, then the abscissa of convergence is a singularity of the LHS. Each $H_{w_{i}}$ is entire, so the first real singularity occurs at -2 . As in (a), this gives a contradiction.

Proof of Theorem A. By Lemma 4.5(b), if $k>1$, (††) cannot be satisfied (for then $\left|\mu_{r}\right|<1$ for all $r$ ). So, for $\pi$ to be increasing, we require $k=1$; i.e. $\tilde{Q}_{p}(z)=1+q(p) z$. Hence $\mu_{1}=-q(p)$ and (††) holds if and only if $s_{n}\left(\mu_{1}\right)=s_{n}(-q(p)) \leq 0$ for $n \geq 2$. By (a) of Lemma 4.5, this only happens if $q(p)=0$ or -1 . Thus

$$
\hat{N}(s)=\zeta(s) \prod_{p \mid P}\left(1+\frac{q(p)}{p^{s}}\right)=\zeta(s) \prod_{i=1}^{l}\left(1-\frac{1}{p_{i}^{s}}\right)
$$

for some prime divisors $p_{1}, \ldots, p_{l}$ of $P$.

\section{Outer g-prime systems with $N(x)-c x$ periodic}

The condition in Theorem 4.4 does not allow us to determine which coefficients $a_{n}$ will lead to outer g-prime systems as they are only necessary and not sufficient. Instead we use the relation

$$
k q\left(p^{k}\right)=\sum_{r=1}^{k} r t\left(p^{r}\right) q\left(p^{k-r}\right)
$$

which follows directly from $Q=e^{T}$. This allows us to calculate $t\left(p^{k}\right)$ explicitly in special cases. Suppose $\tilde{Q}_{p}$ has degree 1. Then $q\left(p^{r}\right)=0$ for $r>1$ and (4.12) gives $k t\left(p^{k}\right)=-(k-1) t\left(p^{k-1}\right) q(p)$ for $k \geq 2$. Thus

$$
t\left(p^{k}\right)=\frac{(-1)^{k-1} q(p)^{k}}{k} .
$$

As a result, $(*)$ holds if and only if $(-q(p))^{k} \leq 1$ for all $k$, which is easily seen to be equivalent to $-1 \leq q(p) \leq 1$ for all $p \mid P$ (i.e. $0 \leq a_{p} \leq 2$ ). In particular, we have proven:

\section{Theorem C}

Let $N \in T$ be such that $N(x)-c x$ has squarefree period $P$. Then $N$ determines an outer g-prime system if and only if

$$
N(x)=\sum_{d \mid P} q(d)\left[\frac{x}{d}\right]
$$

where $q(\cdot)$ is multiplicative, $q(p) \in[-1,1]$, and $c=\prod_{p \mid P}(1+q(p) / p)$.

For example, the outer g-prime systems for which $N(x)-c x$ has period 6 are given by

$$
N(x)=[x]+\lambda\left[\frac{x}{2}\right]+\mu\left[\frac{x}{3}\right]+\lambda \mu\left[\frac{x}{6}\right],
$$

where $(\lambda, \mu \in[-1,1])$ and $(1+\lambda / 2)(1+\mu / 3)=c$. 


\section{References}

[1] T. M. Apostol, Mathematical Analysis, Addison-Wesley, Massachusetts, 1973.

[2] P. T. Bateman and H. G. Diamond, Asymptotic distribution of Beurling's generalised prime numbers in: Studies in Number Theory 6, Prentice-Hall, 1969, pp. 152-212.

[3] P. T. Bateman and H. G. Diamond, Analytic Number Theory, World Scientific Publishing, 2004.

[4] A. Beurling, Analyse de la loi asymptotique de la distribution des nombres premiers généralisés, I, Acta Math. 68 (1937), 255-291.

[5] H. G. Diamond, Asymptotic distribution of Beurling's generalized integers, Illinois J. Math. 14 (1970) 12-28.

[6] G. H. Hardy and E. M. Wright, An introduction to the theory of numbers (fifth edition), OUP, 1979.

[7] T. W. Hilberdink, Flows of zeta functions with periodic integrator, J. Théorie des Nombres Bordeaux 23 (2011) 349-363.

[8] E. Saias and A. Weingartner, Zeros of Dirichlet series with periodic coefficients, Acta Arithmetica 140 (2009) 335-344.

[9] E. C. Titchmarsh, The Theory of Functions, Second edition, Oxford University Press, 1986.

\section{APPENDIX - When does $N(x)=c x+1-c$ determine a g-prime system?}

From the proof of Theorem 2.1 we saw that $\psi^{\prime}(x)=1-x^{-c}$ for $x \geq 1$. Thus $\psi$ (equivalently $\Pi$ ) is increasing for every $c \geq 0$. What about $\pi$ ? Let $\theta=\pi_{L}$ be the generalization of Chebyshev's $\theta$-function. Then $\theta(x)=\sum_{n=1}^{\infty} \mu(n) \psi\left(x^{1 / n}\right)$ so that

$$
\theta^{\prime}(x)=\sum_{n=1}^{\infty} \frac{\mu(n)}{n} x^{\frac{1}{n}-1} \psi^{\prime}\left(x^{\frac{1}{n}}\right)=\frac{1}{x} \sum_{n=1}^{\infty} \frac{\mu(n)}{n}\left(x^{\frac{1}{n}}-x^{\frac{1-c}{n}}\right) .
$$

Let $f$ be the entire function

$$
f(z)=\sum_{n=1}^{\infty} \frac{\mu(n)}{n}\left(e^{\frac{z}{n}}-1\right)=\sum_{k=1}^{\infty} \frac{z^{k}}{k ! \zeta(k+1)} .
$$

Then $e^{x} \theta^{\prime}\left(e^{x}\right)=f(x)-f((1-c) x)$ and $\theta$ is increasing if and only if

$$
f(x) \geq f((1-c) x) \quad \forall x \geq 0 .
$$

For $0 \leq c \leq 2$ this is easily seen to hold as

$$
f(x)-f((1-c) x)=\sum_{k=1}^{\infty} \frac{\left(1-(1-c)^{k}\right) x^{k}}{k ! \zeta(k+1)}
$$

and the coefficients are all non-negative if (and only if) $0 \leq c \leq 2$.

Now consider $c>2$. It is clear that $\left(A_{c}\right)$ holds for all $c>2$ (actually for $c \geq 1$ ) if and only if

$$
f(-x) \leq 0 \quad \text { for } x \geq 0 .
$$

For if $(\mathrm{B})$ is true, then since $(1-c) x \leq 0$, we have

$$
f((1-c) x) \leq 0 \leq f(x)
$$

and $\left(A_{c}\right)$ holds. Conversely, assume $\left(A_{c}\right)$ holds for all $c>2$. Suppose, for a contradiction, that $f\left(-x_{0}\right)>0$ for some $x_{0}>0$. Then

$$
0<f\left(-x_{0}\right)=f\left((1-c) \cdot \frac{x_{0}}{c-1}\right) \leq f\left(\frac{x_{0}}{c-1}\right)
$$

for every $c>2$. This is false for $c$ sufficiently large as the RHS can be arbitrarily close to zero. Thus (B) is true. 
However, we show that $(\mathrm{B})$ is false, and hence that $\left(A_{c}\right)$ fails for some $c>2$.

\section{Theorem A1}

There exists $\lambda>2$ such that for $c \leq \lambda, \pi$ is increasing, while for $c>\lambda, \pi$ is not increasing.

Proof. Clearly, if $\left(A_{c}\right)$ holds for some $c=c_{0}>1$, then it holds for all smaller $c$, since $\left(A_{c}\right)$ is equivalent to

$$
f(-y) \leq f\left(\frac{y}{c-1}\right) \quad \forall y \geq 0
$$

and $f$ is increasing on $(0, \infty)$. Also, if $\left(A_{c}^{\prime}\right)$ holds for all $c<c_{1}$, then by continuity of $f$, it holds for $c=c_{1}$. Now we show $(\mathrm{B})$ is false.

Starting from the formula ${ }^{8} \frac{1}{2 \pi i} \int_{(-1,0)} \Gamma(s) x^{-s} d s=e^{-x}-1(x>0)$ we have

$$
\frac{1}{2 \pi i} \int_{(-1,0)} \frac{\Gamma(s)}{\zeta(1-s)} x^{-s} d s=\sum_{n=1}^{\infty} \frac{\mu(n)}{n} \cdot \frac{1}{2 \pi i} \int_{(-1,0)} \Gamma(s)\left(\frac{x}{n}\right)^{-s} d s=\sum_{n=1}^{\infty} \frac{\mu(n)}{n}\left(e^{-x / n}-1\right)=f(-x),
$$

using the absolute and uniform convergence of the Dirichlet series for $1 / \zeta(1-s)$. Changing the variable gives

$$
f(-x)=\frac{1}{2 \pi i} \int_{(1,2)} \frac{\Gamma(1-s)}{\zeta(s)} x^{s-1} d s
$$

By Mellin inversion

$$
\frac{\Gamma(1-s)}{\zeta(s)}=\int_{0}^{\infty} \frac{f(-x)}{x^{s}} d x
$$

Hence

$$
\int_{1}^{\infty} \frac{f(-x)}{x^{s}} d x=\frac{\Gamma(1-s)}{\zeta(s)}-\int_{0}^{1} \frac{f(-x)}{x^{s}} d x=\frac{\Gamma(1-s)}{\zeta(s)}+\sum_{k=1}^{\infty} \frac{(-1)^{k-1}}{k ! \zeta(k+1)(k+1-s)} .
$$

Since the LHS converges and is holomorphic in $H_{1}$, the singularities at $2,3,4 \ldots$ on the RHS are all removable, as is the singularity at $s=1$.

Suppose now that $f(-x)$ is ultimately of one sign. Then the abscissa of convergence of the LHS Mellin transform must be a (real) singularity of the function. But the first real singularity occurs at -2 (zero of $\zeta)$. This is a contradiction as there are singularities at the non-trivial zeros of $\zeta$ to the right of this. Thus $f(-x)$ cannot ultimately be of one sign; i.e. $f$ changes sign infinitely often in $(-\infty, 0)$ and has infinitely many zeros here.

Thus $\left(A_{c}^{\prime}\right)$ fails for some $c \geq 2$ and hence all larger $c$. Let $\lambda$ denote the supremum of those $c$ for which $\left(A_{c}^{\prime}\right)$ holds. Thus $\left(A_{c}^{\prime}\right)$ holds for $c \leq \lambda$ and fails for $c>\lambda$.

Finally, $\lambda>2$ since $f\left(\frac{y}{\lambda-1}\right) \geq f(-y)$ for all $y \geq 0$ with equality for some $y>0$ (or $\lambda$ would not be optimal) and this is false for $\lambda=2$.

\footnotetext{
${ }^{8}$ Here $\int_{(\alpha, \beta)}$ means $\lim _{T \rightarrow \infty} \int_{\sigma-i T}^{\sigma+i T}$ for any $\sigma \in(\alpha, \beta)$.
} 